\title{
Jejunal adenocarcinoma manifesting as epididymal mass: a rare case scenario
}

\begin{abstract}
Epididymal tumors are rare lesions which we come across in routine practice. It is also a rare site of solid tumor metastases and mimics the chronic mass forming inflammatory pathologies leading to a diagnostic dilemma. Most of the cases of epididymal metastases are diagnosed incidentally. Most cases of epididymal metastases are from genitourinary and gastrointestinal malignancies. We present a case of a middle-aged male presenting with complaints of pain and palpable lump in left inguino-scrotal region, where ultrasound revealed epididymal mass. The diagnosis of epididymal metastases from jejunal adenocarcinoma was made on the basis of CT.
\end{abstract}

Keywords: epidydimis, adenocarcinoma, meatstases, jejunum
Volume 5 Issue 6 - 2018

\author{
Arjit Agarwal,' Ashutosh Kumar, ${ }^{2}$ Shruti \\ Chandak' \\ 'Department of Radiodiagnosis, Teerthanker Mahaveer \\ University, India \\ ${ }^{2}$ Department of Pathology, Teerthanker Mahaveer University, \\ India

\begin{abstract}
Correspondence: Arjit Agarwal,Assistant Professor Department of Radiodiagnosis, Teerthanker Mahaveer Medical College \& Research Centre, Teerthanker Mahaveer University, Moradabad, India, Tel +91-9012209194, Email arjet123456@gmail.com
\end{abstract}

Received: November 07, 2017| Published: November 21, 2018

\section{Introduction}

Epididymal mass is a diagnostic dilemma mostly because of its rare presentation and appearance similar to mass-forming epididymitis. Primary mass of the epidydimal origin are mostly benign and usually confines to the epididymis or spermatic cord. The sonographic appearances of these tumors are non-specific. Metastases to epididymis and spermatic cord accounts for less than $10 \%$ of the malignant cases. ${ }^{1}$ Small bowel adenocarcinoma is one of the rarest primary tumors manifesting as epididymal mass. Here we present a case of primary jejunal adenocarcinoma metastasizing to epididymis.

\section{Case presentation}

A young middle-aged male, presented to the surgical out-patient department with complaint of scrotal fullness and hard palpable lump in left inguino-scrotal region visible from last 15 days. Evaluation by high resolution ultrasonography (HRUS) was advised by the attending clinician.

HRUS of the scrotal and inguinal regions revealed a mild tender, large well-defined near-homogenous mass in the head of left epididymis, measuring approximately $5.0 \times 4.5 \times 4.0 \mathrm{cms}$. The testes appeared to be adhered to the soft tissue mass and floating in the fluid filled scrotal sac (Figure 1). No remarkable vascularity was noted in the mass. No satellite nodule was seen in bilateral inguinoscrotal compartment. Right testes and epididymis were unremarkable. Since there was no clinical complaint related to chest and abdomen, possibility of primary epidydymal tumor was kept with differential diagnoses of mass-forming epididymitis. An old chest radiograph and abdominal ultrasound report were also found to be within normal limits.

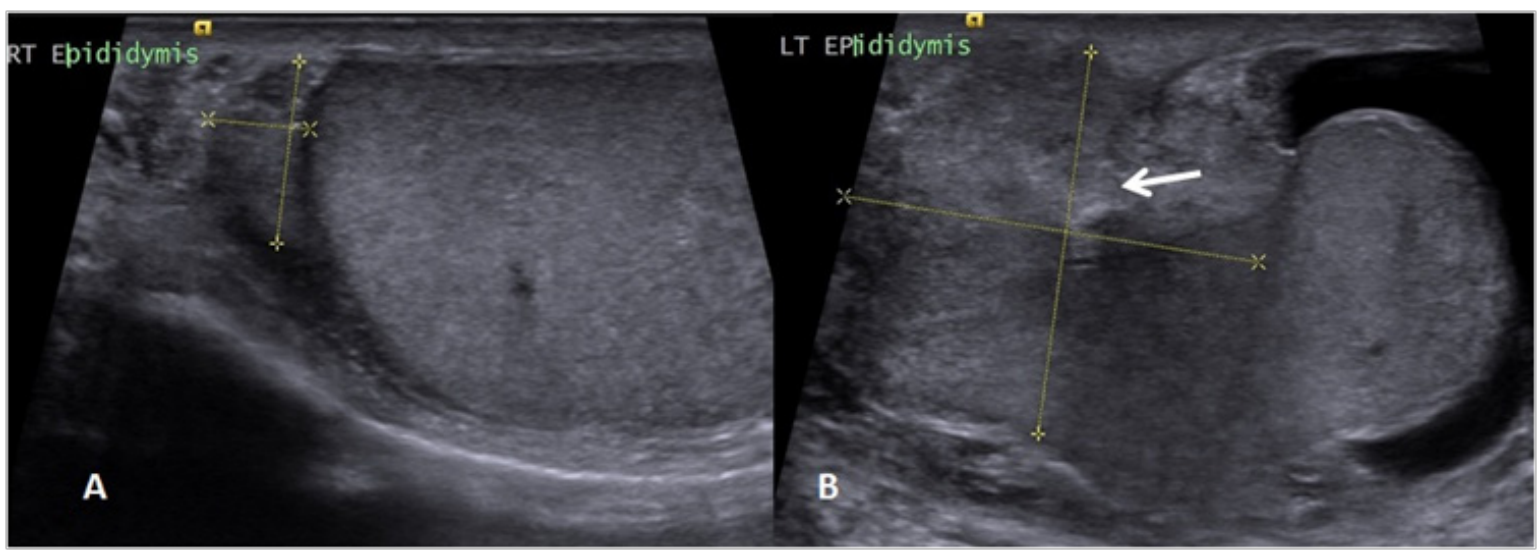

Figure I High resolution USG of scrotum showing normal size and echotexture of right testes and epididymis (B) a large soft tissue mass involving left epididymis and rete testes (white arrow), surrounded by fluid in the scrotal sac.

The patient underwent fine-need aspiration cytology from the epididymal mass which turned out inconclusive. Surgical resection with scrotal exploration was offered to the patient where excision of the mass with left orchiectomy was done. Gross pathology showed a lobulated brown coloured mass involving epididymis head, body and rete testis. No gross infiltration of the testes was found. 
Spermatic cord was edematous without any nodule. Histopathological examination revealed glandular morphology with pleomorphism strongly suggested possibility of metastases from adenocarcinomas (Figure 2). Immunohistochemistry was not performed. Abdominal contrast computed tomography (CT) scan was done which showed a large, non-obstructing heterogenous mass from mid-jejunal origin associated with few enhancing peritoneal and liver surface deposits (Figure 3). A metastatic nodule was also found in visualized section of right lower lung lobe, confirming the diagnoses of metastatic jejunal adenocarcinoma. Due to advanced stage of small-bowel adenocarcinoma, the patient was referred to higher centre for further treatment \& management.

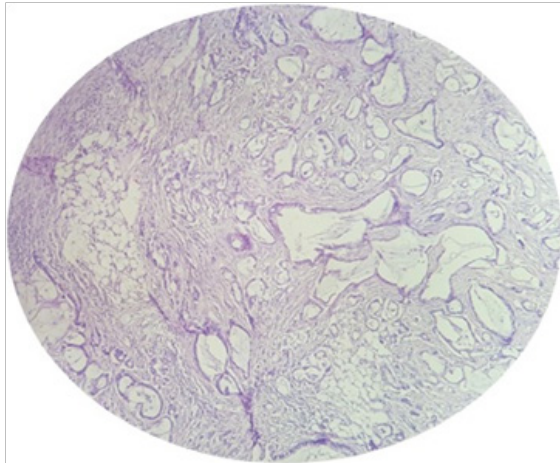

A

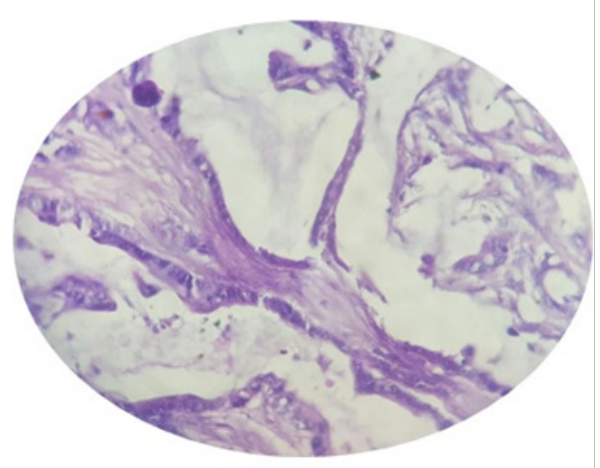

B

Figure 2 Histomorphology (Hematoxylin \& Eosin stain) of epididymal mass showing features of adenocarcinomas. (A) low magnification showing irregularly sized glands lined by malignant epithelial cells with plenty of mucin surrounded by desmoplastic stroma (B) higher magnification showing cells with pleomorphic hyperchromatic nuclei with moderate cytoplasm.

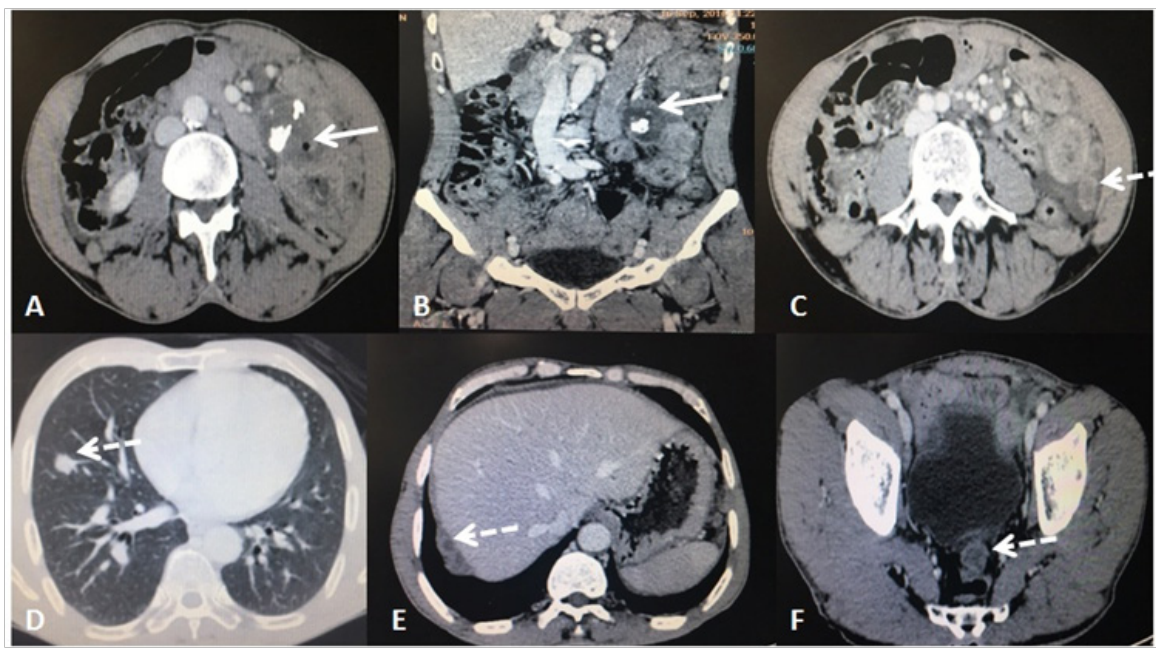

Figure 3 Contrast CT of whole abdomen. (A \& B) Axial and Coronal sections showing heterogenous, necrotic mass arising from jejunum (white arrows) with calcifications. (C to F) Axial sections of abdomen and lower chest showing enhancing nodular deposits (dashed- hite arrows) in left parocolic region (C); right lower lung lobe (D); surface of Liver (E) and recto-vesical space (F).

\section{Discussion}

The most common inguino-scrotal masses are confined to the testicular origin with predominance of infective etiology. Primary epididymal masses are rare where the most common being the adenomatoid tumor of the epididymis. Metastasis to spermatic cord and epididymis is a rare but known phenomenon where the most frequent primary sites are from carcinoma of the stomach and prostate accounting for $42.8 \%$ and $28.5 \%$, respectively. ${ }^{1}$ A previous study analyzed a cohort of 54 Japanese patients showing intra-scrotal metastases and the most frequent site of primary malignancy was found to be the stomach, probably due to the regional dominance of the gastric malignancy. ${ }^{2,3}$ In another study, prostatic carcinomas were found to be the most common primary tumours metastasizing to epididymis with incidence of $37 \%$ and mostly of epithelial origin. ${ }^{4}$

Metastatic nodules in testes account for 3.6\% while that in the paratesticular tissue being $8.1 \%$. Age related distribution shows the predominance of leukaemia and small round cell tumours in childhood, solid masses like adenocarcinomas in middle aged and lymphomas in older man. ${ }^{5,6}$ Solitary metastatic nodule in epididymis is rare and is reported previously from a case of cholangiocarcinoma, however; they are usually a manifestation of disseminated disease as seen in the present case. ${ }^{7}$

Literature search shows that a first case of metastates to epididymis was reported from primary renal cell carcinoma and later from a case of pancreatic malignancy during 1920s. ${ }^{4}$ From then onwards there are many more cases reported but most of them found incidentally on autopsy studies. Apart from prostatic and gastric malignancy, the other primary sites can be kidneys, colon, ileum and pancreas. No case in the literature was found to be from jejunal site as primary malignancy. The spread of metastases from gastrointestinal malignancies is haematogenous while from prostatic carcinomas is direct through vas deferens. 
The primary modality for investigating inguino-scrotal masses is ultrasonography, however; the clinical acumen is also important to exclude other possibilities. The sonographic appearances of the masses are not pathognomic for any entity and most cases mimic mass-forming organized infections. Thus, any testicular or paratesticular nodule should be given a possibility of dissemination of any systemic or organ-specific malignancy and needs to be evaluated under this assumption. As the clinical examination is incomplete without examination of external genitalia, similarly inguino-scrotal ultrasounds with masses should be combined with abdominal ultrasound screening. Targeted surgical intervention can be done in such cases of disseminated malignancies where orchiectomy may resolve the local symptoms and confirm the diagnoses.

\section{Conclusion}

Epididymal tumors are rare and may mislead the clinician and radiologist due to their non-specific appearance. They should be evaluated keeping in mind the possibility of metastases from unknown primary which can prevent unnecessary and over-enthusiastic intervention.

\section{Acknowledgements}

None

\section{Conflict of interest}

The authors declare that there is no conflict of interest.

\section{References}

1. Valizadeh N, Oskuie AE, Tehranchi A. Spermatic cord metastasis as early manifestation of small bowel adenocarcinoma. Urol Ann. 2013;5:53-55.

2. Kanno K, Ohwada S, Nakamura S, et al. Epididymis metastasis from colon carcinoma: A case report and a review of the Japanese literature. Jpn J Clin Oncol. 1994;24(6):340-344.

3. Amin MB. Selected other problematic testicular and paratesticular lesions: rete testis neoplasms and pseudotumours, mesothelial lesions and secondary tumours. Mod Pathol. 2005;18:S131-S145.

4. Powell BL, Craig JB, Muss HB. Secondary malignancies of the penis and epididymis: a case report and review of the literature. J Clin Oncol. 1985;3(1):100-116.

5. Patel SR, Richardson RL, Kvols L. Metastatic cancer to the testes: a report of 20 cases and review of the literature. J Urol. 1989;142:10031005 .

6. Algaba F, Santaularia JM, Villavicencio H. Metastatic tumor of the epididymis and spermatic cord. Eur Urol. 1983;9:56-59.

7. Bennet VS, Bailey DM. Cholangiocarcinoma presenting as a solitary epididymal metastasis: a case report and review of the literature. Diagn Pathol. 2007;2:33. 